\title{
ALGUNAS CONSIDERACIONES BIOLOGICAS DEL TUCUNARE, Cichla ocellaris Schneider
}

\author{
(*) ALCANTARA BOCANEGRA F. \\ (*) GUERRA FLORES H.
}

\section{RESUMEN}

Durante la ejecución del crucero $8508\left(^{(*)}\right.$ del BIC "Rosendo M elo", se efectuaron registros de longitud, peso, grado de llenura del estómago y estadio de madurez sexual de 65 ejemplares de tucunaré, Cichla ocellaris Schneider, capturados en el área de la Reserva Nacional Pacaya-Samiria, por el método de remoción total con rotenona.

La relación peso-longitud de la población estudiada responde a la ecuación W $=a^{n}{ }^{n}$, siendo el crecimiento de tipo alométrico, de acuerdo a Rickér 3(1975), y diferencial para cada sexo a longitudes mayores de $32 \mathrm{~cm}$. La distribución de frecuencias del factor de condición muestra que al estado adulto, los machos presentan una mejor condición que las hembras.

El tamaño mínimo de primera maduración, determinado según SANTOS (1978) y VAZZOLER (1981), es hembras $36 \mathrm{~cm}$. y machos $42 \mathrm{~cm}$. El tamaño al cual el $100 \%$ de individuos alcanzan la madurez, determinado según los investigadores antes citados, es: hembras $44 \mathrm{~cm}$. y machos $50 \mathrm{~cm}$.

Se observó una proporción de sexos de 2: 1 a favor de los machos, verificándose mediante el test del ji cuadrado, a un nivel de probabilidad mayor que 0.5. Sin embargo, la proporción observada puede ser también el resultado de un sesgo introducido en el muestreo.

El $54 \%$ de la población muestreada presentó estómago vacío y el 31\% estomago semi vacío, siendo el $15 \%$ restante semilleno y lleno.

El $45 \%$ de la población muestreada presentó estadio de madurez sexual III 0 maduro y el $38 \%$ II o en maduración, perteneciendo el 17\% restante al 10 inmaduro.

Palabras claves: Relación peso-longitud. Factor de condición.Tamaño mínimo de primera maduración. Tucunaré, Cichla ocellaris.

(*) Instituto de Investigaciones de la A mazonía Peruana IIA P

(*) Efectuado en el mes de agosto de 1985 


\section{SUM M ARY}

On $8580\left(^{*}\right)$ BIC' "Rosendo M elo" cruise, records of length, wight, degree of stomach's fulness and sexual maturity stage in 65 "Tucunare" samples, Cichla ocellaris Shneider were made.

They were captured in the $N$ ational Reserve of Pacaya and Samiria by total removing with rotenone. The wight- lentgth relationship of the examined population ans wers $W=a^{n}$ equation. The growth was alometrica 1 (Richer, 19 75) and diffe rential for each sex. The length w as superior t $032 \mathrm{~cm}$. Distribution of condition fact or frequenci es shows that la adults males present better conditions than females.

According to SANTOS (1978) and VAZZOLER (1981) the minimum size of first maturity is: $36 \mathrm{~cm}$. in females and $42 \mathrm{~cm}$. in males. They established that the size obtained by $100 \%$ of mature individuals is: $44 \mathrm{~cm}$. in fermales and $50 \mathrm{~cm}$. in males.

2:1 sex proportion in favour of males was observed. It was verified by Ji square test to a probability superior 05 . However, the observed proportion can also be the result of and introduced spur in the sample.

$54 \%$ of the sampled population presented and empty stomach, $31 \%$ semi empty stomach and the remainder $15 \%$ a semifilled or a fil led one.

$45 \%$ of the sampled population presented III sexual maturity or mature and $38 \%$ II or the process of maturation and the remaining $17 \%$ I or inamature state.

\section{INTRODUCCION}

El tucunaré, Cichla ocellaris Schneider, es uno de los mayores cíclidos nativos que está más ampliamente distribuido en los ambientes lénticos de la amazonía peruana. Por la calidad de su carne, es una especie que tiene demanda preferencial y permanente en el mercado local.

DOURA DO y DAVIES (1978), estudiaron la relación peso-longitud de Cichla ocellaris Schneider, procedentes de los reservorios de Ceará, Brasil. VERANI (1980), efectuó el cultivo de Sarotherodon niloticus con Cichla ocellaris, en las proporciones 3:1, 6:1, 9:1, 12: 1 y 15: 1; reportando que la optimización de los índices de rendimiento en biomasa acumulada se verificó en la proporción de 9: 1, entre $S$. niloticus y $C$. ocellaris, con valores muy próximos a los optimizados, en la proporción de 6: 1.

Cuanto mas se conozcan los aspectos biológicos de una especie en particular, mejores serán las posibilidades de manejo y regulación de su pesquería. En tal sentido, el presente trabajo es una contribución al mejor conocimiento biológico de la especie y en él se reporta la relación peso-longitud y el factor de condición, el tamaño 
mínimo de primera maduración y el tamaño al cual el $100 \%$ de los individuos son sexualmente maduros; así como la proporción de sexos y algunos aspectos de su nutrición y maduración sexual.

\section{MATERIAL Y METODOS}

Con ocasión del crucero 8508 del BIC "Rosendo M elo" a la cuenca del bajo U cayali, se efectuaron capturas de tucunaré, Cichla ocellaris Schneider, mediante el método de remoción total con rotenona, en áreas de la Reserva Nacional Pacaya- Samiria (Cotococha), habiendo sido capturados 65 ejemplares, registrándose su longitud, peso, estadio de repleción del estómago y estadio de madurez sexual.

Se determinó la relación peso-longitud y el factor de condición, de acuerdo a la ecuación general $\mathrm{W}$ aL ${ }^{n}$. Los valores de a y $n$ se determinaron empíricamente, cambiando la relación exponencial $W \mathrm{aL}^{\mathrm{n}}$ en una relación lineal, usando logaritmos y calculando la línea de regresión por el método de los cuadrados mínimos (LAGLER, 1971; BERGER, 1972; RICKER，1975; BAGENAL, 1978; WOSNITZA， 1978).

La línea de regresión y los pesos calculados se obtuvieron usando una calculadora Texas Instrument TI $58 \mathrm{C}$, programable. Para cada clase de longitud se efectuó el cálculo del factor de condición, utilizando el peso promedio empírico en la relación

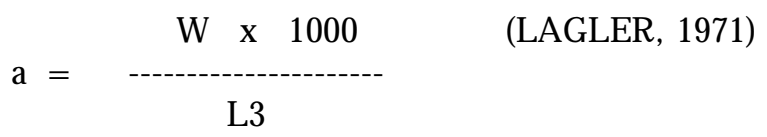

Se analizó la distribución de frecuencia relativa de machos y hembras, determinándose las longitudes de primera maduración sexual y aquellas en que todos los individuos de la población están aptos a participar del proceso reproductivo, de acuerdo a SANTOS (1978) y VAZZOLER (1980) (Figura 3).

Se verificó la proporción de sexos aplicando la prueba del ji cuadrado $\left(\mathrm{X}^{2}\right)$, según LEWIS (1970), ? (0 - E²/E) (Tabla 2)

Se consideraron cuatro grados de llenura del estómago, de acuerdo a LOZANO (1970): I vacio; II semi vacio; III semi Ileno; IV Ileno (Tabla 3)

Además se consideraron tres estadios de madurez sexual: I = inmaduro; II = en maduración y $\mathrm{III}=$ maduro. Se clasificaron como estadios de madurez $\mathrm{I}$, cuando las gónadas de machos y hembras eran de aspecto filamentoso, con escasa 
vascularización, pegadas a la columna, transparentes y sin visualización de óvulos. En estadio II, se consideraron las gónadas mas desarrolladas que el estadio anterior, con vascularización notoria en machos y hembras, siendo de color blanquecino en tos machos y presentando óvulos visibles a simple vista, de color amarillo en las hembras. En el estadio III, se consideraron gónadas bien desarrolladas, con óvulos visibles a simple vista, en diferentes fases de desarrollo y presencia de óvulos maduros listos para la puesta y caracterizados por su color amarillo grisáceo y mayor desarrollo; los testículos de este estadio presentaron color blanco, con presencia de abundante esperma (Tabla 4).

\section{RESULTADOS}

$L$ a relación peso-longitud de la población estudiada responde a la ecuación exponencial $W=a L^{n}$, siendo $W=0.009956 L^{3.138822}$, la ecuación que describe la relación para los machos y $\mathrm{W}=0.014149 \mathrm{~L}^{3.035660}$, para las hembras. En ambos casos, el valor del exponente $n$ es mayor a 3, indicando que la especie presenta un crecimiento de tipo alométrico, de acuerdo a RICKER (1975). A longitudes mayores que $32 \mathrm{~cm}$., se observa un crecimiento diferencial para cada sexo, verificándose alta adherencia de las observaciones empíricas a las curvas de ajustamiento (Figura 1). El factor de condición observado presentó una ligera diferencia en relación al sexo. L as hembras presentaron un valor medio de $16.0 \mathrm{~cm}$. en tanto que los machos presentaron un valor de 16.4 (Tabla 1 ) (*)

(*) Sin embargo, segun la distribución de frecuencia de este factor, se observa que las hembras presentan mejor condición hasta longitudes de $34 \mathrm{~cm}$. y a partir de esta longitud, los machos muestran una mejor condición. (Figura 2).

\section{DISCUSION}

DOURADO y DAVIS (1978), estudiaron la relación peso-longitud de Cichla ocellaris Schneider, procedentes de los reservorios de Ceará, Brasil, analizando los sexos en conjunto; sin embargo, esta especie presenta un notable dimorfismo sexual al estado adulto, siendo los machos, entre otros aspectos más robustos que las hembras. (Tabla 1; Figura 1)

Según SANTOS (1978), un problema básico consiste en la determinación del tamaño mínimo a partir del cual el animal comienza a reproducirse. Si bien no hay un tamaño fijo a partir del cual los individuos comienza a reproducirse, la frecuencia relativa aumenta grandemente con el tamaño. En este caso, Santos define como tamaño de primera maduración, al correspondiente a la frecuencia de 0.5 (50\%). 
Este análisis permite, además, la estimación de la longitud total media en que todos los individuos de la población (100\%) están aptos a participar activamente del proceso reproductivo (L 100\%) VAZZOLER (1981).

Por otro lado, según VAZZOLER (1981), para la estimación de la longitud media (o edad) de inicio de la primera maduración, se colectan datos sobre el estadio de madurez sexual de la especie durante un ciclo completo (periodo entre dos desoves).

En el caso del tucunaré, $C$. ocellaris, Schneider, por tratarse da una especie que se reproduce a través de todo el año y debido a la elevada incidencia de individuos adulto, se analizaron los datos de una sola captura, determinándose la longitud de primera maduración para las hembras es de $36 \mathrm{~cm}$, mientras que para los machos es de $42 \mathrm{~cm}$.; asimismo, la longitud a la cual el $100 \%$ de individuos son maduros es de $44 \mathrm{~cm}$. para las hembras y d $50 \mathrm{~cm}$. para los machos (Figura 3).

De un total de 65 ejemplares, se observaron 42 machos y 23 hembras, lo que da una proporción de sexos de 18: 1; a favor de los machos. Al aplicar la prueba del ji cuadrado $\left(X^{2}\right)$ a la proporción 2: 1, se obtuvo una probabilidad de ocurrencia mayor de 0.5 , que corrobora las observaciones de campo (Tabla 2). Sin embargo, la proporción observada puede ser también el resultado de un sesgo introducido en el momento de recoger los especimenes, ya que los peces mayores atraen más la atención y estos, en su mayoría son machos, por lo que son necesarios estudios posteriores en este sentido.

En cuanto al grado de llenura del estómago, el 54\% de la población muestreada ( $n=65$ ) presentó el estómago vacío y el 31\% estómago semi vacío, de donde se infiere que los valores del factor de condición, calculados en el presente caso, reflejan en gran medida el estado somático de la especie (Tabla 3). En adición a lo anteriormente anotado, el $45 \%$ de la muestra se observó en el estadio de madurez sexual III o maduro y el $38 \%$ en el estadio II o en maduración (T abla 4).

L as observaciones anteriores indican que la especie se encontraba en un estado de reproducción activa, atenuando su alimentación en este proceso, debido posiblemente a la protección de su prole. ya que, según FONTENELE (1955) citado por VERANI (1980), tanto macho como hembras protegen sus huevos y su prole hasta que las larvas alcanzan aproximadamente $35 \mathrm{~cm}$. de longitud. 
TABLA 1

RALACION-PESO LONGITUD EN GRAMOS Y CENTIMETROS DE Cichla ocellaris, Schneider

\section{HEMBRAS}

Longi-

Condici.
Rango en Peso Mínimo
Promedio Peso Factor $\mathrm{N}^{\mathrm{o}}$ Máximo Empírico Calculado Condición

\section{MACHOS}

Rango en Peso Mínimo
Promedio Peso Factor de

Máximo Empírico Calculado

\begin{tabular}{llllllllllllllc}
\hline 22 & - & - & - & - & - & 168 & - & 1 & - & 180 & - & 180 & 163 & 15.0 \\
24 & - & - & - & - & - & 219 & - & - & - & - & - & - & 214 & - \\
26 & 1 & - & 260 & - & 260 & 279 & 14.7 & - & - & - & - & - & 275 & - \\
28 & 3 & 340 & - & 380 & 363 & 350 & 16.5 & 2 & 330 & - & 350 & 340 & 347 & 15.5 \\
30 & - & - & - & - & - & 431 & - & 3 & 400 & - & 453 & 425 & 431 & 15.7 \\
32 & 5 & 423 & - & 586 & 512 & 524 & 15.6 & 3 & 463 & - & 510 & 481 & 528 & 14.7 \\
34 & 3 & 630 & - & 727 & 672 & 630 & 17.0 & 1 & - & 660 & - & 660 & 638 & 16.8 \\
36 & 2 & 720 & - & 740 & 730 & 750 & 15.6 & 1 & - & 840 & - & 840 & 764 & 18.0 \\
38 & 1 & - & 920 & - & 920 & 884 & 16.7 & 2 & 1000 & - & 1020 & 1010 & 905 & 18.0 \\
40 & 5 & 800 & - & 1120 & 1024 & 1032 & 16.0 & 7 & 1000 & - & 1170 & 1074 & 1063 & 16.8 \\
42 & 3 & 1180 & - & 1230 & 1206 & 1198 & 16.2 & 7 & 1150 & - & 1329 & 1220 & 1239 & 16.4 \\
44 & - & - & - & - & - & 1379 & - & 7 & 1350 & - & 1620 & 1451 & 1434 & 17.0 \\
46 & - & - & - & - & - & - & - & 6 & 1510 & - & 1670 & 1598 & 1649 & 16.4 \\
48 & - & - & - & - & - & - & - & - & - & - & - & - & 1884 & - \\
50 & - & - & - & - & - & - & - & 2 & 1860 & - & 2250 & 2055 & 2142 & 16.4 \\
$X$ & - & - & - & - & - & - & 16.0 & - & - & - & - & - & - & 16.4 \\
Rang0 & - & - & - & - & - & $14-7$ & 17.0 & - & - & - & - & - & 14.7 & 18.0
\end{tabular}




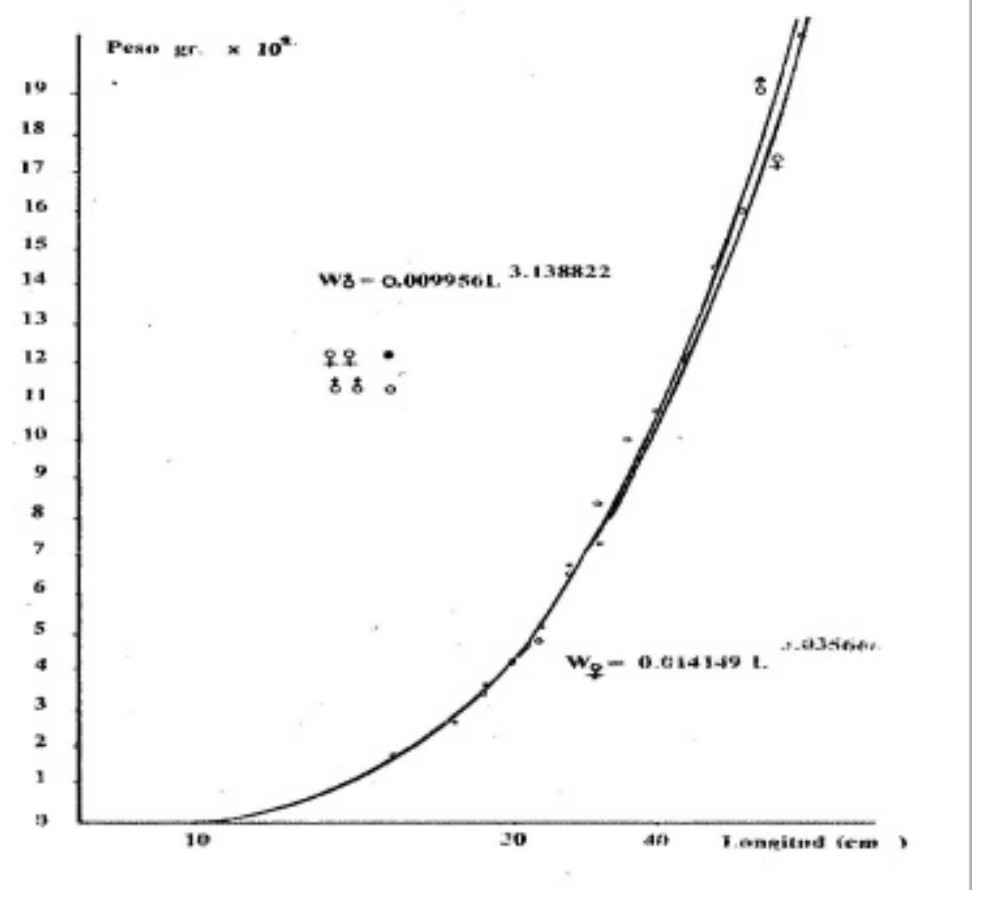

FIGURA 1. RELACION ENTRE LA LONGITUD TOTAL Y EL PESO DE Cichla ocellaris Schneider 


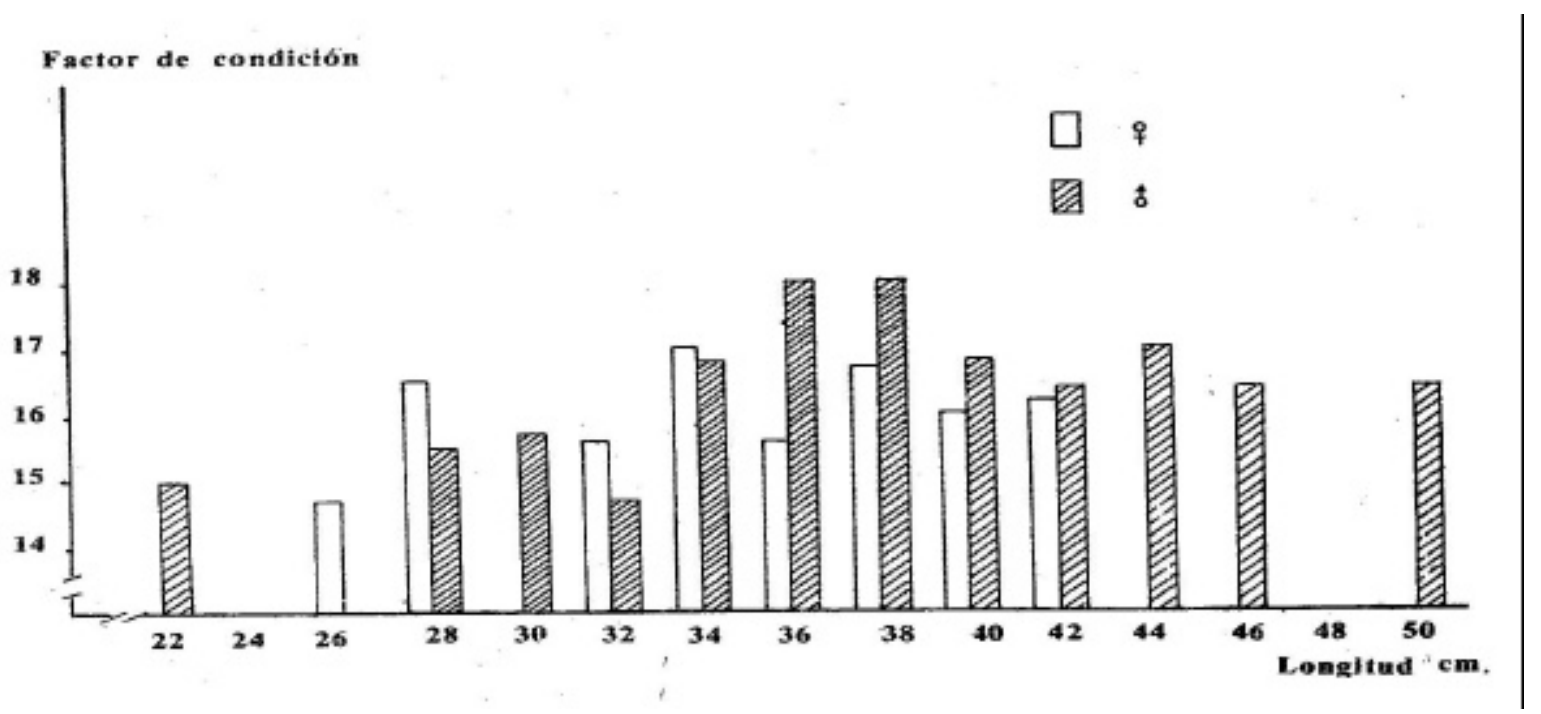

FIGURA 2. DISTRIBUCION DE FRECUENCIA DEL FACTOR DE CONDICION DE Cichla ocellaris Achneider POR SEXO Y LONGITUD. 
TABLA 2. PRUEBA JI CUADRADO PARA LA PROPORION DE SEXOS 2:1 Cichla ocellaris Schneider

\begin{tabular}{|c|c|c|c|}
\hline CLASE & Fo & Fe & D \\
\hline M achos & 42 & 43.3 & -1.3 \\
\hline Hembras & 23 & 21.7 & +1.3 \\
\hline (Total) & $(65)$ & $(65.0)$ & $(0.0)$ \\
\hline
\end{tabular}

$$
\begin{aligned}
& 2 \\
& X_{c}=\begin{array}{rc}
(-1.3)^{2} & (+1.3)^{2} \\
43.3 & 21.7
\end{array} \\
& X_{c}^{2}=0.1169 \quad \text { con 1g. 1. donde } P>0.5 \\
& \text { Fo = Frecuencia observada } \\
& \mathrm{Fe} \quad=\text { Frecuencia esperada } \\
& \text { D } \quad=\text { Desviación }
\end{aligned}
$$




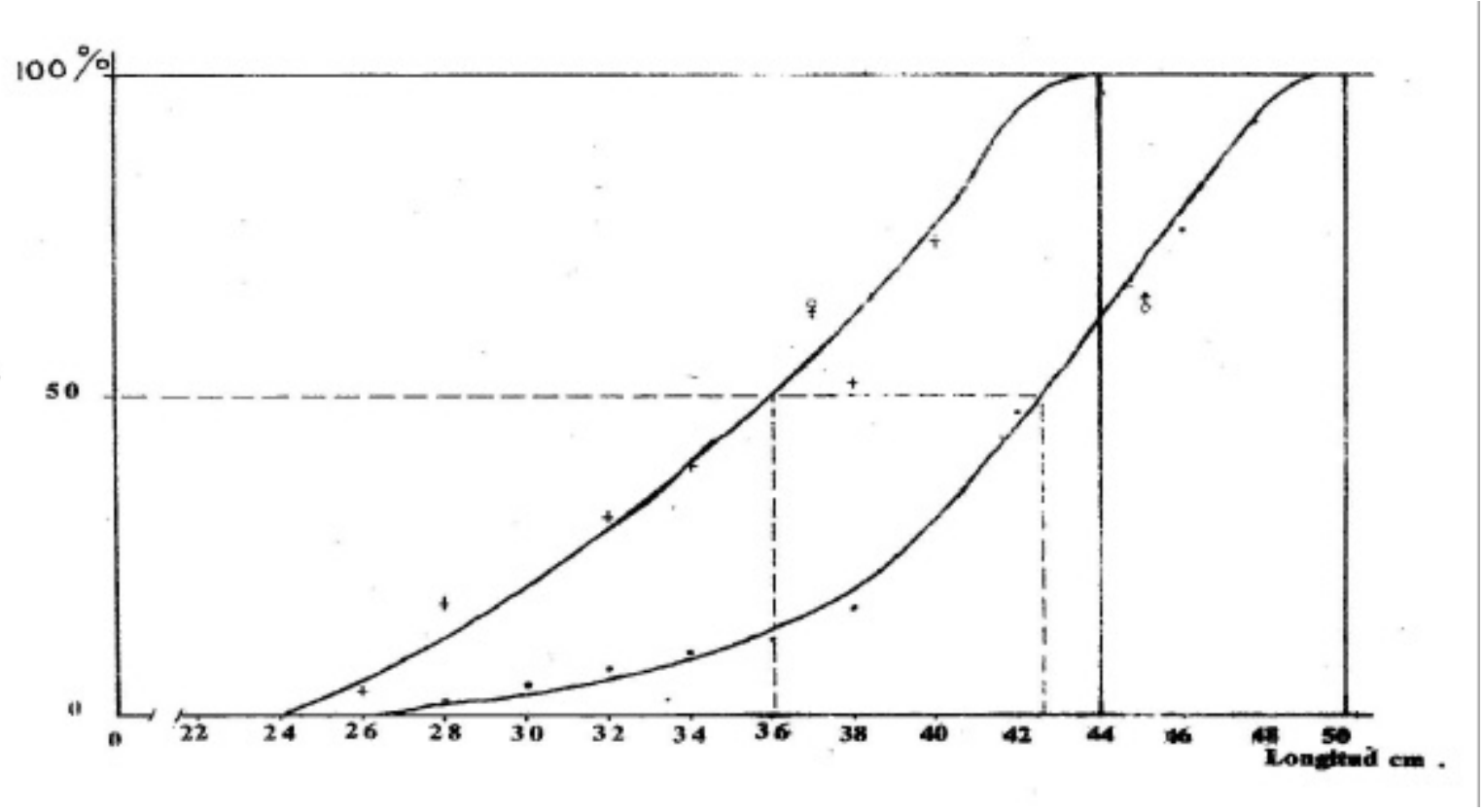

FIGURA 3. DISTRIBUCION DE FRECUENCIA RELATIVA DE ADULTOS DE Cichla ocellaris Schneider POR CLASES DE LONGITUD. 
TABLA 3. GRADO DE LLENURA DEL ESTOMAGO DE Cichla ocellaris Schneider.

\begin{tabular}{lcc}
\hline CLASES & FRECUENCIA & $\%$ \\
\hline Vacio & 35 & 54 \\
Semi vacio & 20 & 31 \\
Semi lleno & 3 & 4 \\
Lleno & 7 & 11 \\
\hline Total & 65 & 100 \\
\hline
\end{tabular}

TABLA 4. ESTADIO DE MADUREZ SEXUAL DE Cichla ocellaris Schneider

ESTADIO

I ( Inmaduro)

II ( En maduración)

III ( M aduro)

Total
FRECUENCIA

11

25

29

65
$\%$

17

38

45 


\section{REFERENCIAS BIBLIOGRAFICAS}

BAGENAL, B.T. and E W. TES CII. 1978. Age and Growth-The lenght Weight relationship. In $M$ ethods for $A$ ssessment of Fish Production in Fresh $W$ aters. 3rd. ed. 1. B. P. Handbooks No 3. Blackwell. Scientific Publications. Oxford. pp. 126.128.

BERGER, C. 1972. Estudio del factor de condición del "paiche". Tesis Bach. Cienc. Biol. U.N.M.S.M. Lima-Perú. pp 3-8.

DOURADO, F. O. and W. D. DAVIES. 1978. Lenght weight Relationships and Condition Indices of Fishes from Reservoirs of Ceará, Brasil. Research and Development Series No 18. International Center for Aquaculture. Auburn University. 5pp.

LAGLER K. F. 1971. Fresh Water Fishery Biology. Second Edition. W. M. C. Brown Company Publishers. Eleventh Printing. Dubuque, lowa. pp. 163-165.

LEWIS, A. E. 1970. Bioestadística. ira. Edición. 2da. impresión. Compañía Editorial Continental S. A. M éxico. pp. 145-161.

LOZAN O, C. F. 1970. Oceanografía, Biología Marina y Pesca. Tomo 1, Ed. Paraninfo. M adrid. España. p. 311.

RICKER, W. E. 1975. Computation and Interpretation of Biological Statistics of Fish Populations. Bull. Fish. Res. Board Can. 191. pp. 209-210.

SA NTOS, E. P. DOS. 1978. Dinamica de populacoes aplicada a pesca e piscicultura. Sao Paulo. HUCITEC. Ed. da Universidade de Sao Paulo. p. 23.

VAZZOLER, A.E. de M. 1981. Manual de métodos para estudos biológicos de populacoes de peixes; reproducao e crecimiento. Brasilia, CNPq. Programa Nacional de Zoología pp. 29.36, 63-64.

VERANI. J. R. 1980. Control populacional em cultivo intensivo consorciado entre a tilapia do Nilo, Sarotherodon nilo ticus Linnaeus, 1757 e o Tucunaré común, Cichla ocellaris Schneider 1801. A spectos cuantitativos. Tesis para obtencao do titulo de $\mathrm{M}$ estre em Ecología e Recursos $\mathrm{N}$ aturais. U niversidade F ederal de Sao Carlos- SP. pp. 21, 71-74. 
WOSNITZA, C.; F. A. DAVILA, 1978. M anual de dinámica de poblaciones de peces. Universidad Nacional de Trujillo. Departamento de Ciencias Biológicas. Trujillo - Perú. pp. 13-15. 